\title{
Polymer-based flexible and multi-directional tactile sensor with multiple NiCr piezoresistors
}

\author{
Soonjae Pyo ${ }^{1 \dagger}$, Jae-Ik Lee ${ }^{2 \dagger}$, Min-Ook Kim ${ }^{1}$, Hyung-Kew Lee ${ }^{3}$ and Jongbaeg Kim ${ }^{1 *}$
}

\begin{abstract}
A polymer-based tactile sensor with flexibility and multi-directional sensing capability is presented. The proposed sensor consists of a polydimethylsiloxane (PDMS) bump, a polyimide (PI) substrate, $\mathrm{Cr} / \mathrm{Au}$ electrode lines for electrical connection, NiCr piezoresistors, and an SU-8 support structure. The sensing mechanism is based on piezoresistive effect, in which the resistance of $\mathrm{NiCr}$ changes under mechanical load. The PMDS bump positioned at the center of the sensor transfers an applied force to the PI film, and the piezoresistors are differently deformed depending on the magnitude and direction of the force. A diaphragm structure formed by the SU-8 support with a trench allows the piezoresistor to be effectively deformed. Simulation and experimental results confirm that magnitude and direction can be obtained from an arbitrarily applied force by comparing the change in resistance of each sensing element. Based on its compatibility with conventional microfabrication, the proposed sensor may be a promising candidate for a low-cost tactile sensing solution for human-machine interfaces.
\end{abstract}

Keywords: Tactile sensor, Strain gauge, Flexibility, Multi-directional force sensor, Polymer micromachining

\section{Introduction}

Tactile interfaces are attracting significant attention in the field of robotics because they can realize the force feedback control of robots [1]. A tactile sensor that acquires information via physical contact is a key element of a tactile interface. In particular, miniaturized tactile sensors have been actively developed owing to their potential for use in medical applications, such as robotassisted surgery systems and cancer diagnosis [2, 3]. For such applications, tactile sensors are supposed to consist of soft and flexible biocompatible materials in order to prevent organ damage and to allow the sensors to be easily mounted on a complex-shaped robot hand. Multidirectional sensing capability over a wide range of forces is also an essential feature for precise and stable measurement. To meet these requirements, a variety of flexible tactile sensors based on various sensing mechanisms have been developed, including resistive [4], capacitive [5], and piezoelectric [6] types.

\footnotetext{
*Correspondence: kimjb@yonsei.ac.kr

†Soonjae Pyo and Jae-lk Lee contributed equally to this work

1 School of Mechanical Engineering, Yonsei University, 50 Yonsei-ro,

Seodaemun-gu, Seoul 03722, Republic of Korea

Full list of author information is available at the end of the article
}

When compared with other sensing mechanisms, resistive sensors have advantages such as simple device structure and ease of signal processing on the acquired data [7]. In recent years, coupled with progress in the scalable production of nanomaterials, flexible resistive tactile sensors utilizing mainly conductive-nanomaterial-polymer composites have been studied [8-10]. To date, however, most previous works have focused on the development of tactile sensors only for normal force detection, with very few studies on multi-directional force sensing [11-13]. Moreover, nanomaterials tend to agglomerate in uncured viscous polymers, making it difficult to uniformly disperse them in the polymer matrix. This leads to performance deviation between fabricated devices, which limits the reproducibility of the sensor. One promising alternative is to integrate a thin-film piezoresistor into a polymer substrate [14, 15]. For example, Hwang et al. [14] demonstrated a flexible tactile sensor based on thin metal strain gauges that were patterned on a polymer substrate, and both normal/shear load detection and reproducibility were achieved. Nevertheless, the extraction of magnitude and direction from an arbitrarily applied force is challenging because the output signal of the sensor is asymmetrical to the direction of the shear force. In addition, 
an elastomer substrate with energy dissipation through material damping limits the deformations of the piezoresistors, which results in sensitivity reduction [16].

In this study, we report a diaphragm-like tactile sensor utilizing multiple piezoresistor configurations that are capable of detecting multi-directional forces. The proposed polymer-based sensor is batch-fabricated on a 4 -in. $\mathrm{SiO}_{2} / \mathrm{Si}$ wafer via conventional microfabrication to demonstrate its practical use. The dependence of the deformation of piezoresistors on the direction of the applied force was thoroughly investigated by finite element analysis (FEA). To verify the sensing performance of the fabricated device, changes in the resistances of the piezoresistors for normal and shear forces were measured. Based on the piezoresistive characteristics, both the magnitude and direction of the applied force were successfully detected.

\section{Proposed concept and design}

The schematics of the polymer-based tactile sensor are illustrated in Fig. 1. The main structure of the proposed sensor is composed of a polydimethylsiloxane (PDMS) bump, a polyimide (PI) substrate, and an SU-8 support. The PDMS bump is utilized to effectively transfer multi-directional input forces to the piezoresistors [6], while the SU-8 support forms a diaphragm structure with a trench. As shown in the bottom, four $\mathrm{NiCr}$ thin films and double-layered $\mathrm{Cr} / \mathrm{Au}$ electrode lines are patterned on the PI substrate. Amongst the many piezoresistive materials available, we used $\mathrm{NiCr}$ alloy as a sensing element because its low temperature coefficient of resistance minimizes the thermal effect on force-sensing performance [15]. Figure 2 shows the sensing mechanism of the presented sensor. The fundamental principle is based on the piezoresistive effect where the resistance of the piezoresistor changes under mechanical load. Generally, the resistance of the piezoresistor decreases and increases with respect to compressive and tensile stresses, respectively (Fig. 2a). When force is applied to the bump positioned at the center of the four sensing elements, each element is deformed differently depending on the magnitude and direction of the force (Fig. 2b). For example, a normal force induces identical compressive stress on the four sensing elements, and thus the resistance of all of the piezoresistors decreases equally. On the other hand, under a shear force in the $\mathrm{x}$-direction, tensile and compressive stresses are applied to sensing element 1 $\left(E_{1}\right)$ and $3\left(E_{3}\right)$, respectively, which causes the resistance
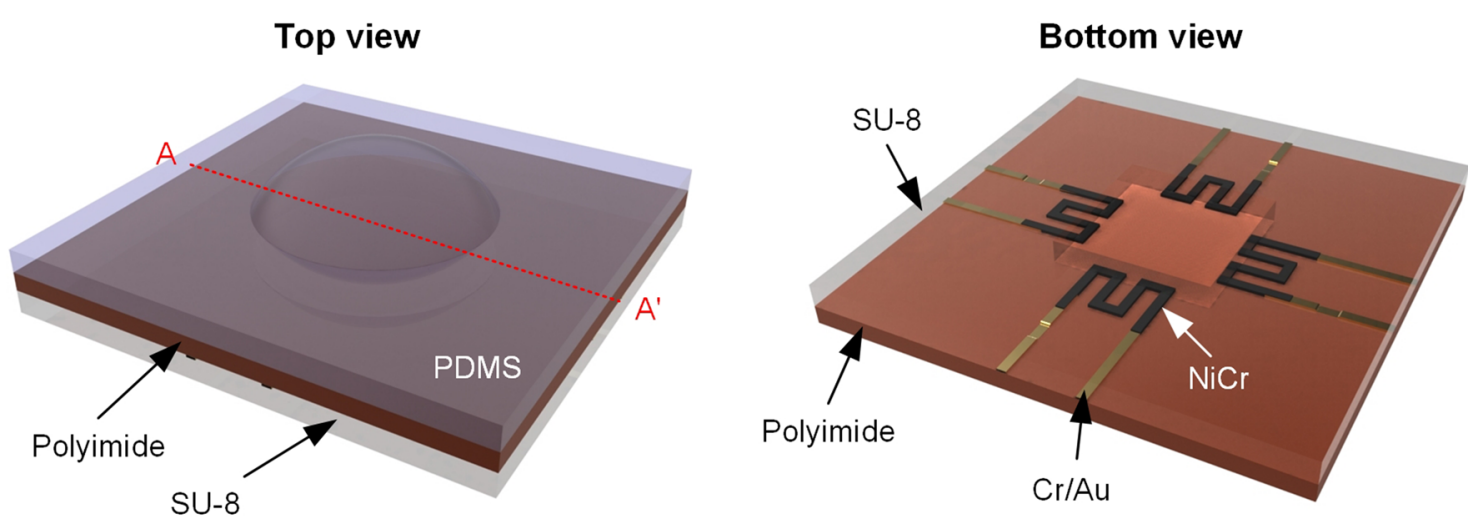

Cross-sectional view

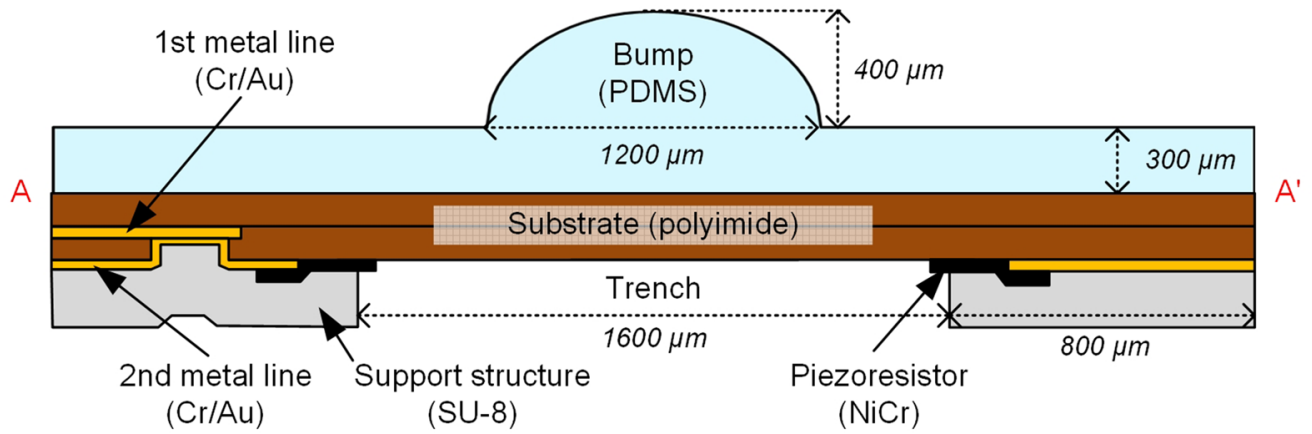

Fig. 1 Schematics of the polymer-based flexible tactile sensor with integrated multiple piezoresistors. The main structure is comprised of flexible and biocompatible materials including PDMS, PI, and SU-8. The patterned $\mathrm{NiCr}$ and double-layered $\mathrm{Cr} / \mathrm{Au}$ lines serve as piezoresistor and electrode, respectively 
a

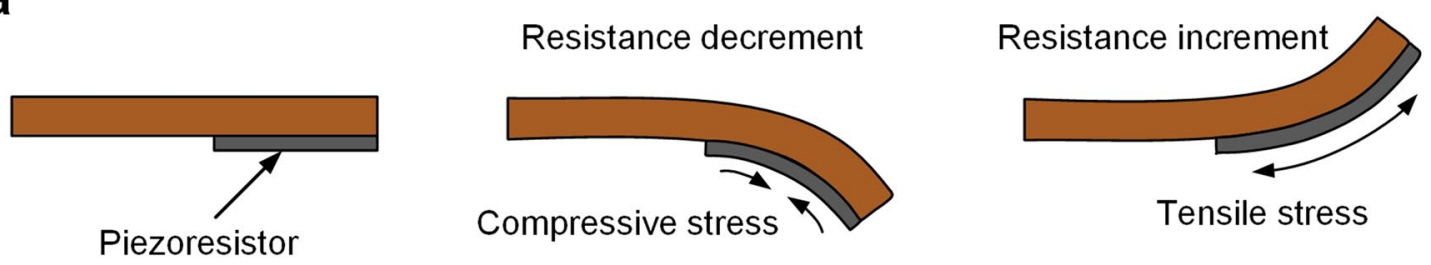

b
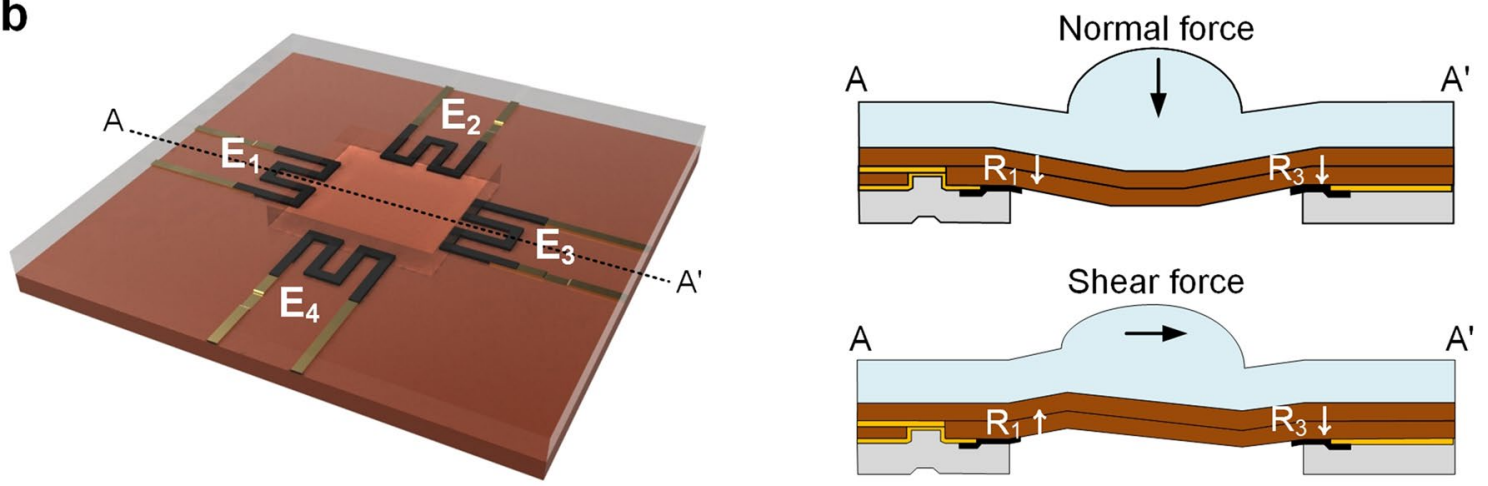

Fig. 2 Sensing mechanism of the proposed multi-directional tactile sensor. a Change in electrical resistance of piezoresistor with respect to applied stress. Compressive and tensile stresses cause decrease and increase in the resistance of the piezoresistor, respectively. $\mathbf{b}$ Operating principle for normal and shear force detections. The resistances of the four piezoresistors change identically under a normal force. In contrast, when a shear force is applied, the resistance of each piezoresistor changes differently

of $E_{1}$ to increase and that of $E_{3}$ to decrease. According to these characteristics, we can distinguish magnitude and direction by comparing the resistance of each piezoresistor [17].

\section{Fabrication}

Figure 3 shows the fabrication process for the polymerbased flexible tactile sensor, including the patterning of piezoresistors and metal lines, and the assembly of the main structure and the PMDS bump layer. The PDMS layer was fabricated separately and attached to the surface of the PI substrate. First, a $20 \mu \mathrm{m}$-thick PI (PI-1388, Vtec) was spin-coated onto a $\mathrm{SiO}_{2} / \mathrm{Si}$ wafer and dried at $325{ }^{\circ} \mathrm{C}$ on a hot plate for $20 \mathrm{~min}$ (Fig. 3a). In order to define the first electrode lines, a positive photoresist (AZ1512, Microchemicals) was patterned via photolithography. The $\mathrm{Cr} / \mathrm{Au}$ layer (20/200 nm) was subsequently patterned using thermal evaporation and a lift-off process (Fig. 3b). After a $2 \mu \mathrm{m}$-thick PI was spin-coated onto the substrate, a $100 \mathrm{~nm}$-thick Al layer was then deposited as a etch mask for the PI patterning because it shows strong etch resistance to oxygen plasma. Next, the second photolithography and Al etching were performed to define via holes for the vertical interconnection between the first and second electrode lines, followed by oxygen plasma treatment for PI etching. After removal of the remaining $\mathrm{Al}$ layer (Fig. 3c), the second electrode lines ( $\mathrm{Cr} / \mathrm{Au}$ layer) were patterned via photolithography, sputtering, and the lift-off process (Fig. 3d). Similarly, NiCr $(80 / 20 \mathrm{wt} \%)$ patterning processes that include photolithography, evaporation, and lift-off were carried out to form piezoresistors (Fig. 3e). For the trench structure, a $100 \mu$ m-thick SU-8 (SU-8 2000, MicroChem) was spin-coated onto the substrate and patterned using photolithography (Fig. 3f). Then, one edge of the wafer was soaked in a buffered hydrofluoric acid for $30 \mathrm{~s}$, and the PI film with sensor components was carefully detached from the wafer (Fig. 3g). The PDMS bump was separately prepared via drop-casting with an $\mathrm{Al}$ mold fabricated by micromilling and then bonded onto the bottom of the PI film (Fig. 3h). Before the bonding, a lowpower oxygen plasma treatment was conducted on the PI surface in order to enhance the adhesion between the PI and the PDMS.

\section{Results and analysis}

The optical microscope images in Fig. 4a show the top and bottom views of the piezoresistor. The serpentine $\mathrm{NiCr}$ piezoresistor is located on the boundary of the diaphragm structure to maximize its deformation under a load. The PDMS bump and metal lines electrically connected with the piezoresistor are also clearly observed. Figure $4 \mathrm{~b}$ presents a photograph of a pixelated $3 \times 3$ tactile sensor array that is batch-fabricated 


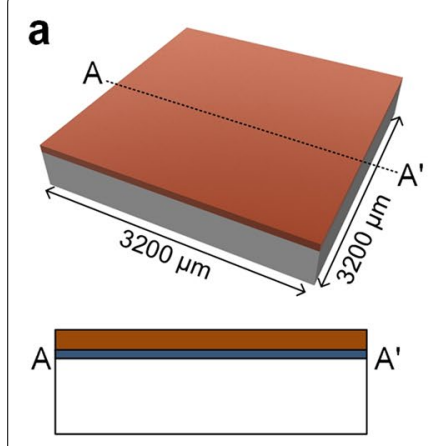

PI spin coating

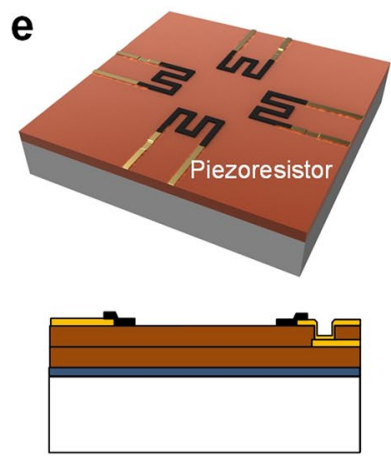

NiCr deposition/patterning

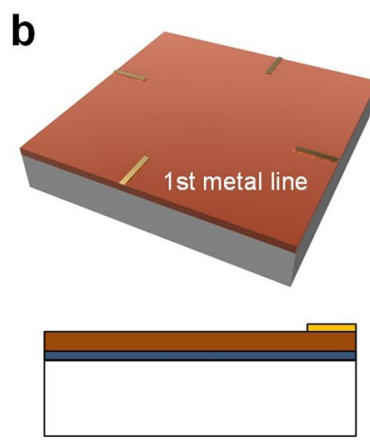

Au deposition/patterning
C

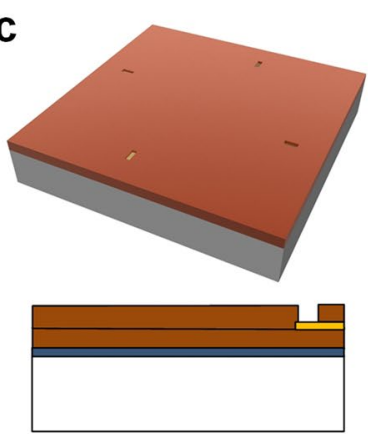

$\mathrm{PI}$ coating/patterning d

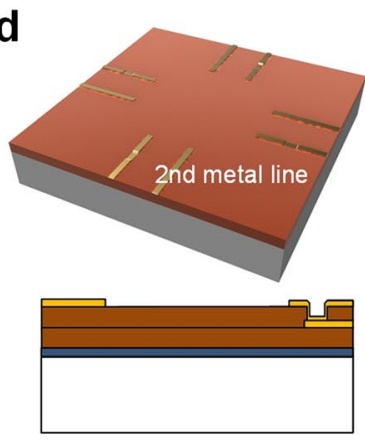

Au deposition/patterning f

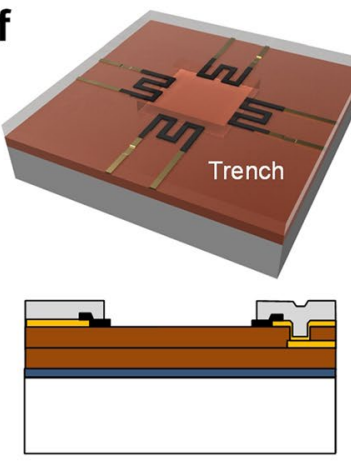

SU-8 coating/patterning g
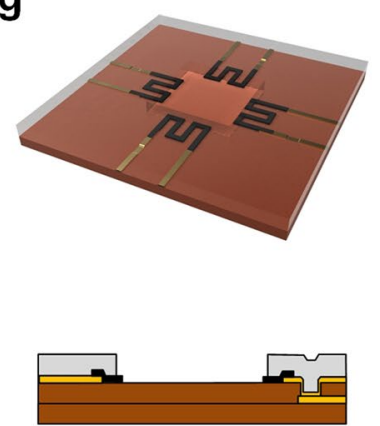

Release h

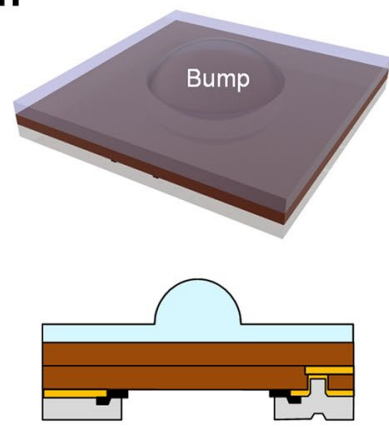

PDMS bonding

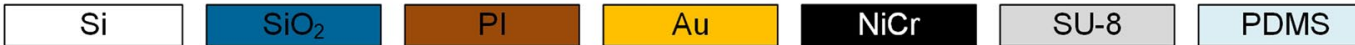

Fig. 3 Fabrication process flow of the polymer-based flexible and multi-directional tactile sensor. NiCr piezoresistors and double-layered metal lines are patterned via conventional microfabrication, including photolithography, evaporation, sputtering, plasma etching, and a lift-off process. Then, the PI film with sensor components is detached from the wafer. Finally, the PDMS bump that is fabricated separately is assembled with the main structure

on the wafer. The sizes of a single sensor and a trench are $3200 \times 3200 \mu \mathrm{m}^{2}$ and $1600 \times 1600 \mu \mathrm{m}^{2}$, respectively. Each sensor can operate independently through separate electrode lines.

In order to verify the proposed concept, the strain generated along the PI surface against the direction of the input force was investigated by FEA (Fig. 5). Threedimensional (3D) modeling and FEA were conducted using a 3D computer-aid design tool (Solid Works 2011) and finite element software (ANSYS 16.2), respectively. The Young's moduli of the PDMS, PI, and SU-8 were set to 15,2600 , and $4400 \mathrm{MPa}$, respectively, with Poisson's ratios of $0.45,0.35$, and 0.22 for PDMS, PI, and SU-8. The boundary condition for the bottom of the SU- 8 support was fixed in all directions, and a force was applied on top of the PDMS bump. Figure 5a shows the FEA result of the strain on the PI surface under a normal load of $10 \mathrm{mN}\left(90^{\circ}\right)$. A strain of $-1.14 \times 10^{-6}$ was generated on the surface where $E_{1}$ and $E_{3}$ were located, which means that their resistance decreased by the same magnitude. In contrast, under a shear force of $10 \mathrm{mN}\left(0^{\circ}\right)$, the strain values on the surface of $E_{1}$ and $E_{3}$ were $0.74 \times 10^{-6}$ and $-0.74 \times 10^{-6}$, respectively (Fig. $5 \mathrm{~b}$ ), implying that resistance of $S_{1}$ decreased while that of $S_{2}$ increased. We also calculated the distribution of strain generated on the PI surface under an oblique force $\left(45^{\circ}\right)$ of $14.14 \mathrm{mN}$, which is the vector sum of normal $(10 \mathrm{mN})$ and shear $(10 \mathrm{mN})$ forces. As shown in Fig. $5 \mathrm{c}$, both strains generated on $\mathrm{E}_{1}\left(-0.41 \times 10^{-6}\right)$ and $\mathrm{E}_{3}\left(-1.88 \times 10^{-6}\right)$ exhibited negative values but different magnitudes. Interestingly, the FEA result was identical to the superposition of the data for normal (Fig. 5a) and shear (Fig. 5b) forces. This indicates that an applied force could easily be separated into normal and shear force components by comparing the responses of the piezoresistors when a force comprised of normal and shear forces is applied.

The force-sensing performance of our polymer-based sensor was evaluated under different loading conditions. 
a

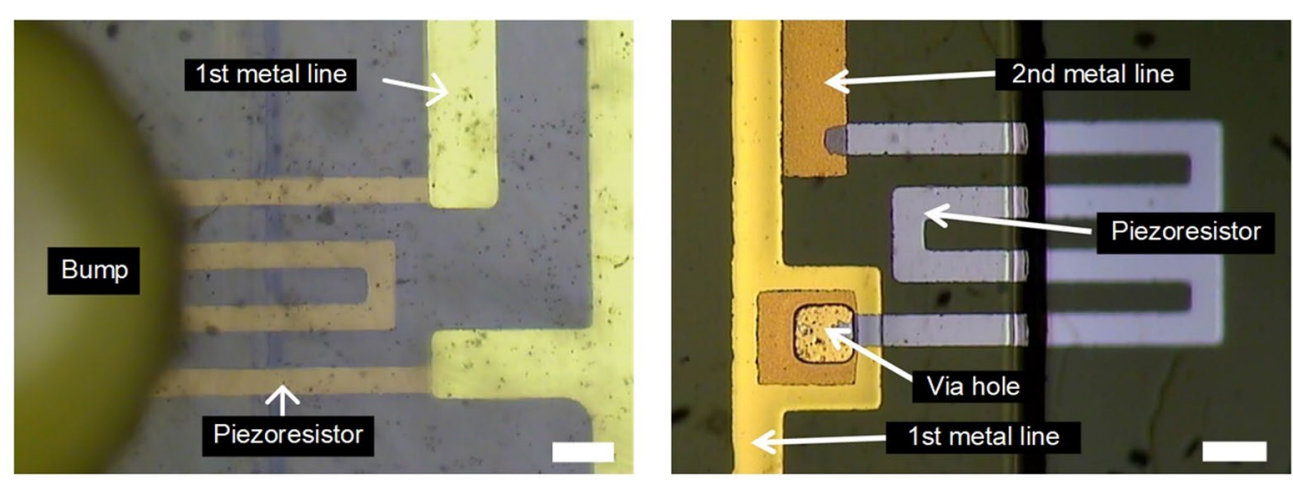

b

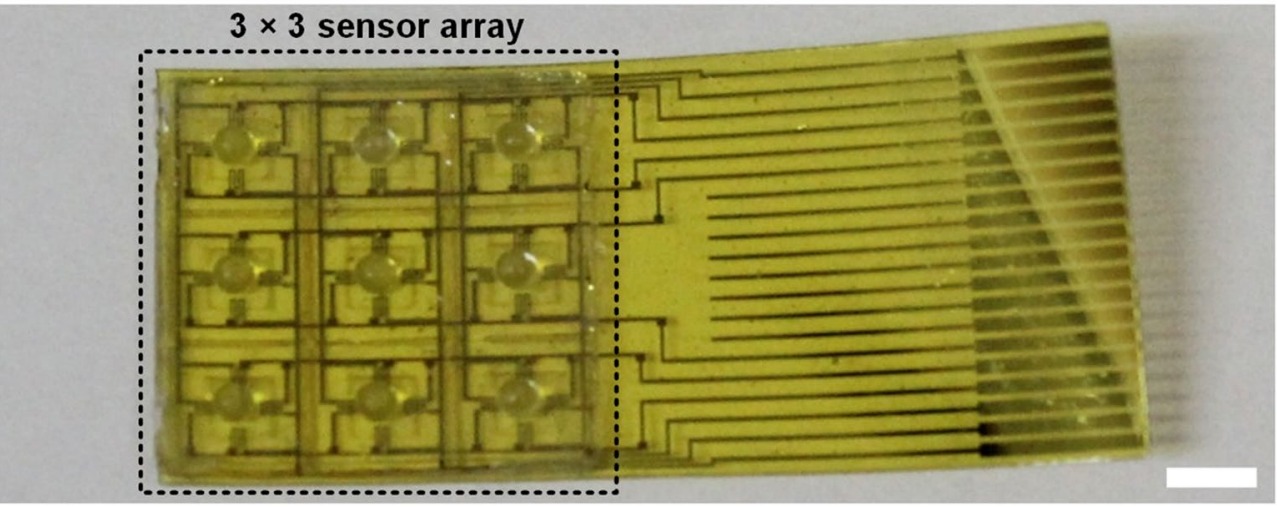

Fig. 4 As-fabricated polymer-based tactile sensor array. a Optical microscope images showing the top and bottom views of the sensor (scale bar: $100 \mu \mathrm{m})$. b Photograph of a pixelated $3 \times 3$ sensor array that can operate independently (scale bar: $2 \mathrm{~mm}$ )
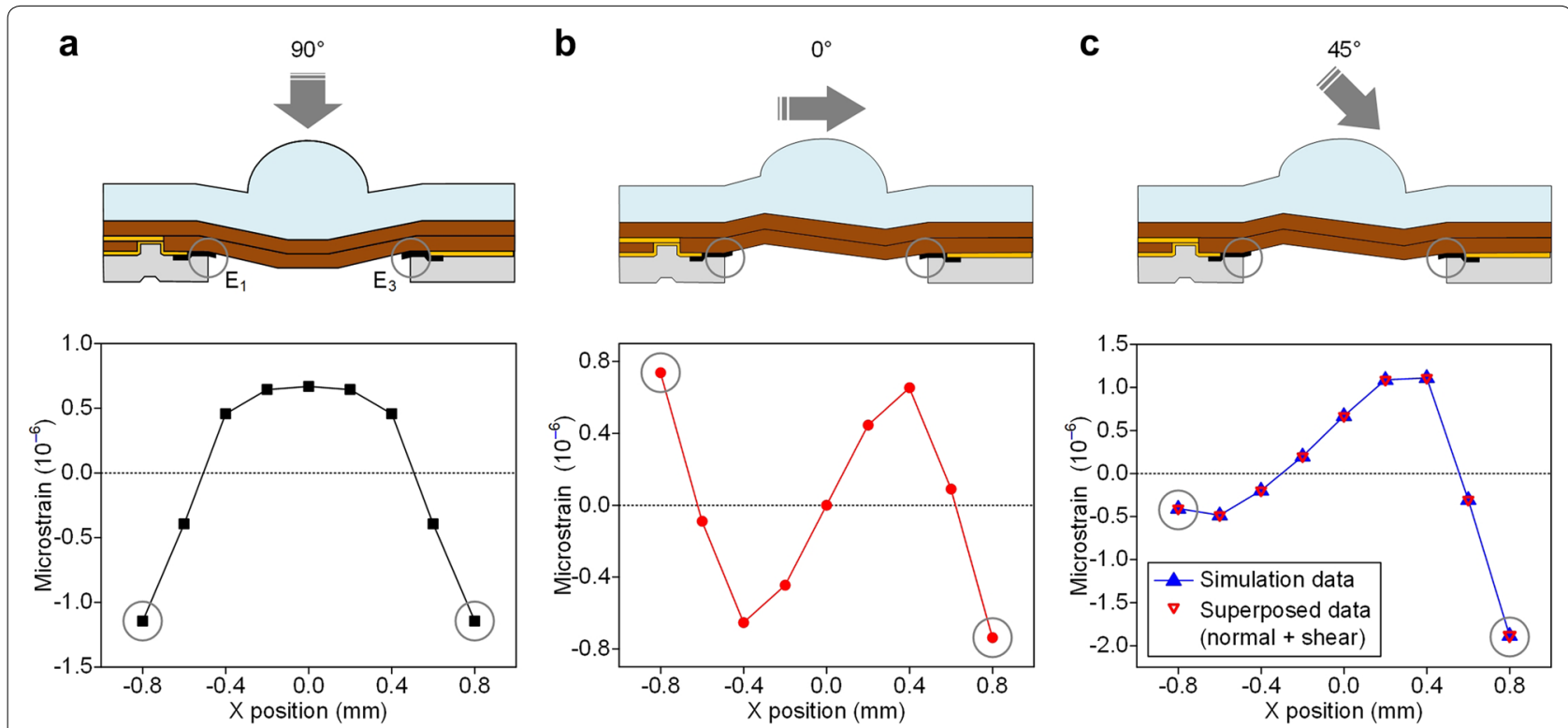

Fig. 5 FEA results for strain generated along the PI surface in response to the input force at $\mathbf{a} 90^{\circ}, \mathbf{b} 0^{\circ}$, and $\mathbf{c} 45^{\circ}$. The strain value depends on the direction of the applied force 
Figure 6 illustrates the schematic of the experimental setup to investigate sensor responses to normal and shear forces. Force was applied using a micromanipulator, and a load cell (CWFS, Bongshin load cell) was employed to precisely measure the magnitude of the applied force. The resistance of each sensing component was measured using a source meter (2400, Keithley) at room temperature in air. The change in the resistance of $\mathrm{S}_{3}$ with respect to normal force up to $500 \mathrm{mN}$ was measured and plotted in Fig. 7a. The tested sensing range of $0-500 \mathrm{mN}$ would be suitable for use in medical applications such as robot-assisted surgery system [18]. The measured initial resistance was approximately $8 \mathrm{k} \Omega$, and the resistance decreased with the increase in applied force because compressive stress was applied
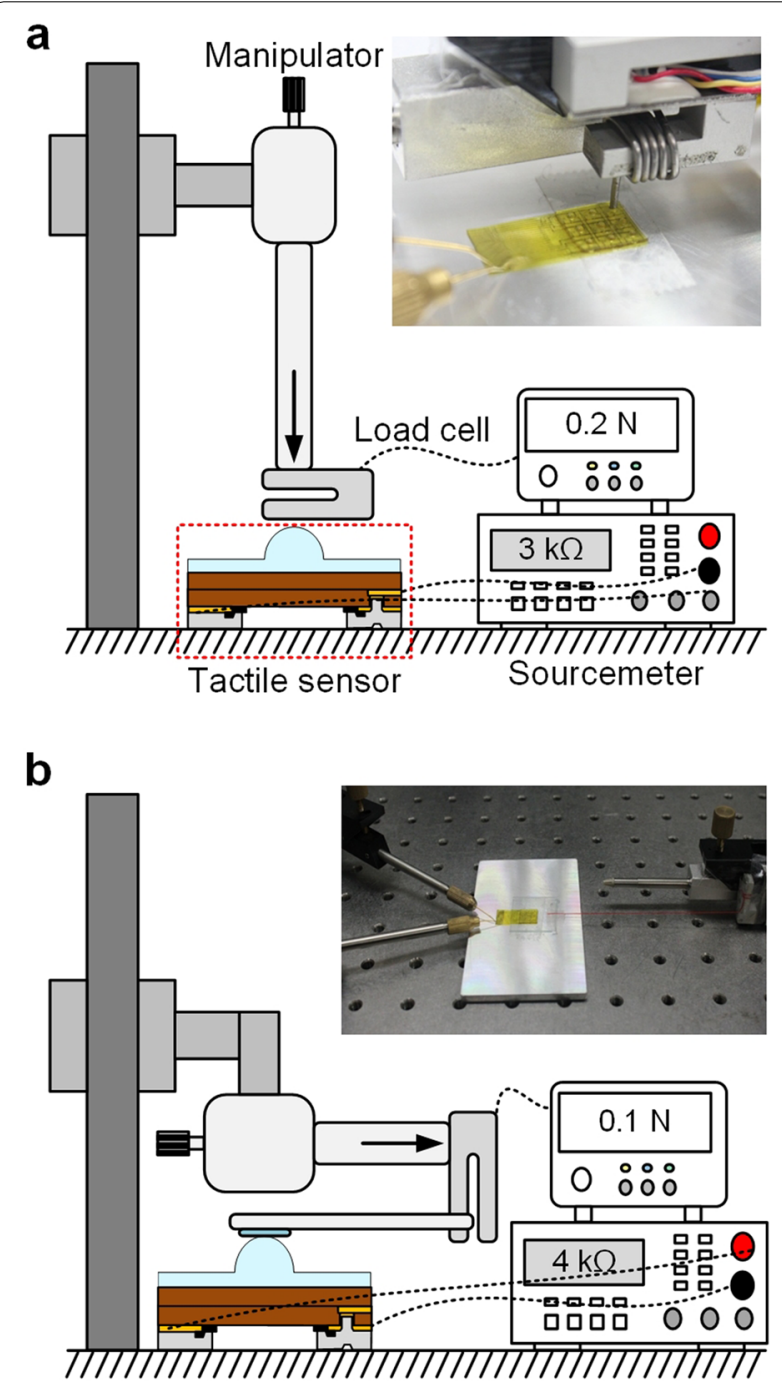

Fig. 6 Detailed schematic and photograph of the experimental setup to explore the force-sensing performance of the tactile sensor for $\mathbf{a}$ normal and $\mathbf{b}$ shear loads to $E_{3}$. Notably, the sensor response could be divided into two regions with different slopes. The slopes were calculated as -4.9 and $-3.6 \Omega \mathrm{mN}^{-1}$ for forces in the ranges of $0-300$ and $300-500 \mathrm{mN}$, respectively. This could be attributed to the fact that the PI film starts to make contact with the ground when a force of $300 \mathrm{mN}$ is applied. Thus, it is expected that the linear sensing range could be widened by increasing the thickness of the SU-8 support structure. Figure $7 \mathrm{~b}$ shows the changes in resistances of $E_{1}$ and $E_{3}$ against applied shear force in the $\mathrm{x}$-direction to the bump. The resistance of $E_{1}$ increased with a slope of $1.2 \Omega \mathrm{mN}^{-1}$, while that of $E_{3}$ decreased with a slope of $-1.2 \Omega \mathrm{mN}^{-1}$. The results indicate that tensile and compressive stresses of the same magnitude are applied to $E_{1}$ and $E_{3}$, respectively, which is consistent with our FEA result (Fig. 5b). This symmetric characteristic to the direction of the shear force is an important feature that enables our sensor to detect magnitude and direction from an arbitrarily applied force, unlike the previous sensor, which exhibited an asymmetric response [14]. When a three-dimensional (3D) force is applied to the bump, changes in the resistance of $\mathrm{E}_{1-4}$ can be expressed as follows:

$$
\begin{aligned}
& \Delta R_{1}=S_{1, \mathrm{x}} \cdot F_{\mathrm{x}}+S_{1, \mathrm{y}} \cdot F_{\mathrm{y}}+S_{1, \mathrm{z}} \cdot F_{\mathrm{z}} \\
& \Delta R_{2}=S_{2, \mathrm{x}} \cdot F_{\mathrm{x}}+S_{2, \mathrm{y}} \cdot F_{\mathrm{y}}+S_{2, \mathrm{z}} \cdot F_{\mathrm{z}} \\
& \Delta R_{3}=S_{3, \mathrm{x}} \cdot F_{\mathrm{x}}+S_{3, \mathrm{y}} \cdot F_{\mathrm{y}}+S_{3, \mathrm{z}} \cdot F_{\mathrm{z}} \\
& \Delta R_{4}=S_{4, \mathrm{x}} \cdot F_{\mathrm{x}}+S_{4, \mathrm{y}} \cdot F_{\mathrm{y}}+S_{4, \mathrm{z}} \cdot F_{\mathrm{z}}
\end{aligned}
$$

where $\Delta R_{\alpha}$ and $S_{\alpha, \beta}$ are the changes in the resistance of $E_{\alpha}$ and slope (resistance change per input force) of $E_{\alpha}$ to the applied force in the $\beta$-direction, respectively. $F_{x}, F_{y}$, and $F_{\mathrm{z}}$ are the $\mathrm{x}-, \mathrm{y}-$, and z-directional components of the applied force, respectively. Based on the FEA and experimental results, the relationship between the slopes of $E_{1-4}$ can be defined as follows:

$$
\begin{aligned}
& S_{1, \mathrm{x}}=-S_{3, \mathrm{x}} \\
& S_{2, \mathrm{y}}=-S_{4, \mathrm{y}} \\
& S_{1, \mathrm{y}}=S_{3, \mathrm{y}} \\
& S_{2, \mathrm{x}}=S_{4, \mathrm{x}} \\
& S_{1, \mathrm{z}}=S_{2, \mathrm{z}}=S_{3, \mathrm{z}}=S_{4, \mathrm{z}}
\end{aligned}
$$

based in Eqs. (1-9), $F_{\mathrm{x}}, F_{\mathrm{y}}$, and $F_{\mathrm{z}}$ can be expressed as follows:

$$
\begin{aligned}
& F_{\mathrm{x}}=\left(\Delta R_{1}-\Delta R_{3}\right) / 2 S_{1, \mathrm{x}} \\
& F_{\mathrm{y}}=\left(\Delta R_{2}-\Delta R_{4}\right) / 2 S_{2, \mathrm{y}} \\
& F_{\mathrm{z}}=\left(\Delta R_{1}+\Delta R_{2}+\Delta R_{3}+\Delta R_{4}\right) / 4 S_{1, \mathrm{z}}
\end{aligned}
$$




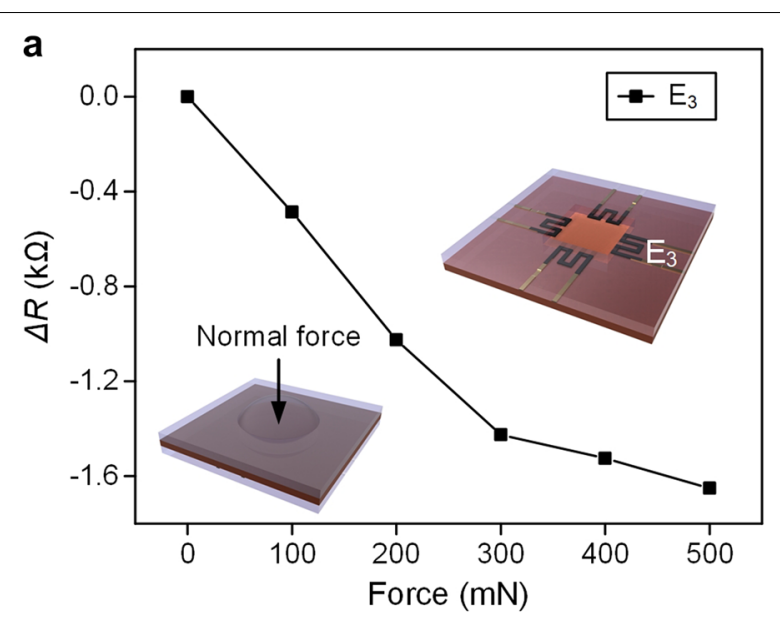

b

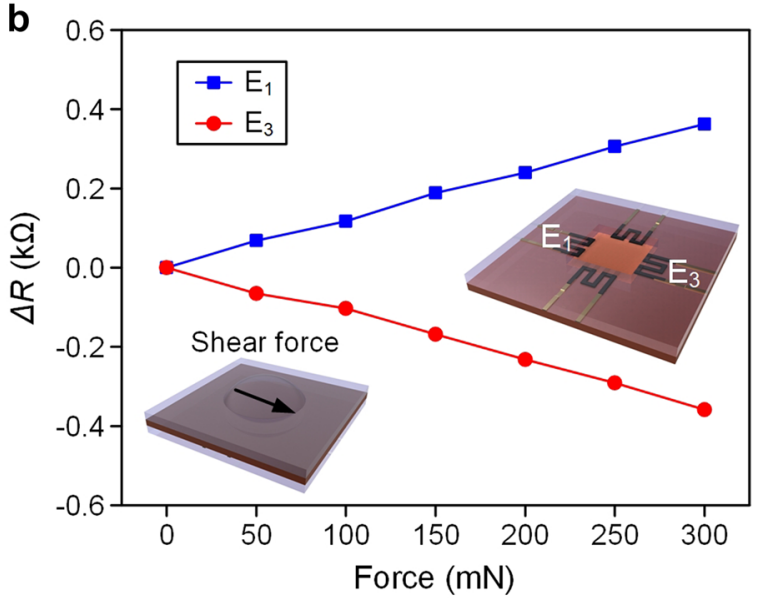

Fig. 7 Changes in resistance of the piezoresistors against applied a normal and $\mathbf{b}$ shear forces. The resistance of $E_{3}$ decreases as the applied normal force increases, with a slope of -4.9 and $-3.6 \Omega$ $\mathrm{mN}^{-1}$ for force ranges of $0-300$ and $300-500 \mathrm{mN}$, respectively. When a shear force in the $x$-direction is applied, the resistance of $E_{1}$ increases with a slope of $1.2 \Omega \mathrm{mN}^{-1}$, while that of $E_{3}$ decreases with a slope of $-1.2 \Omega \mathrm{mN}^{-1}$

It is noteworthy that tactile sensors must be designed to produce linear signals to obtain the magnitudes and directions from multi-directional forces using Eqs. (10-12). Design improvements for linearity over a wide force range and its experimental evaluation are underway and will be provided in a future publication.

\section{Conclusion}

We have demonstrated a flexible and multi-directional tactile sensor composed of polymers, $\mathrm{NiCr}$ piezoresistors, and thin metal electrodes. The proposed sensor array was batch-fabricated through conventional microfabrication including photolithography, evaporation, plasma etching, and molding. The strains generated on the sensing elements under different loading conditions were investigated by FEA, which confirmed that the strain values of each sensing element depended on the direction of the applied force. We also measured the changes in resistance of the piezoresistors for normal and shear forces. The experimental results validated the multi-directional sensing capability of our sensor based on piezoresistive characteristics. Our polymer-based sensor can be used for low-cost tactile sensor applications that require multi-directional sensing and mechanical flexibility.

\section{Acknowledgements \\ Not applicable.}

\section{Authors' contributions}

SP, JIL, HKL and JK developed the idea. SP and JIL carried out fabrication, measurement, and analysis of the results, and wrote the manuscript. MOK performed finite element analysis and supported fabrication process and measurement. HKL suggested fabrication method and material selection. JK supervised the research and reviewed the manuscript. All authors read and approved the final manuscript.

\section{Funding}

This work was supported by the National Research Foundation of Korea (NRF) Grant funded by the Korea government (MSIT) (2018R1A4A1025986).

\section{Availability of data and materials}

All data generated or analysed during this study are included in this published article

\section{Competing interests}

The authors declare that they have no competing interests.

\section{Author details}

${ }^{1}$ School of Mechanical Engineering, Yonsei University, 50 Yonsei-ro, Seodaemun-gu, Seoul 03722, Republic of Korea. ${ }^{2}$ Department of Neurosurgery, Massachusetts General Hospital, Harvard Medical School, 50 Blossom Street, Boston, MA 02114, USA. ${ }^{3}$ Korea Research Institute of Standards and Science, 267 Gajeong-ro, Yuseong-gu, Daejeon 34113, Republic of Korea.

Received: 3 April 2019 Accepted: 23 May 2019

Published online: 27 May 2019

\section{References}

1. Pacchierotti C, Sinclair S, Solazzi M, Frisoli A, Hayward V, Prattichizzo D (2017) Wearable haptic systems for the fingertip and the hand: taxonomy, review, and perspectives. IEEE Trans Haptics 10:580-600

2. Tiwana MI, Redmond SJ, Lovell NH (2012) A review of tactile sensing technologies with applications in biomedical engineering. Sens Actuat A Phys 179:17-31

3. Pacchierotti C, Prattichizzo D, Kuchenbecker KJ (2016) Cutaneous feedback of fingertip deformation and vibration for palpation on robotic surgery. IEEE Trans Biomed Eng 63:278-287

4. Lee Jl, Pyo S, Kim MO, Kim J (2018) Multidirectional flexible force sensors based on confined, self-adjusting carbon nanotube arrays. Nanotechnology 29:055501

5. Lee HK, Chung J, Chang SI, Yoon E (2011) Real-time measurement of the three-axis contact force distribution using a flexible capacitive polymer tactile sensor. J Micromech Microeng 21:035010

6. Kim MO, Pyo S, Oh Y, Kang Y, Cho KH, Choi J, Kim J (2018) Flexible and multi-directional piezoelectric energy harvester for self-powered human motion sensor. Smart Mater Struct 27:035001

7. Maheshwari W, Saraf R (2008) Tactile devices to sense touch on a par with a human finger. Angew Chem Int Ed 47:7808-7826 
8. Pang Y, Tian H, Tao L, Li Y, Wang X, Deng N, Yang Y, Ren TL (2016) Flexible, highly sensitive, and wearable pressure and strain sensors with graphene porous network structure. ACS Appl Mater Interfaces 8:26458-26462

9. Wang T, Zhang Y, Liu Q, Cheng W, Wang X, Pan L, Xu B, Xu H (2018) A selfhealable, highly stretchable, and solution processable conductive and polymer composite for ultrasensitive strain and pressure sensing. Adv Funct Mater 28:1705551

10. Zhao X, Xu W, Yi W, Peng Y (2019) A flexible and highly pressure-sensitive PDMS sponge based on silver nanoparticles decorated reduced graphene oxide composite. Sens Actuat A Phys 291:23-31

11. Hu CF, Su WS, Fang W (2011) Development of patterned carbo nanotubes on a 3D polymer substrate for the flexible tactile sensor application. J Micromech Microeng 21:115012

12. Han JE, Kim D, Yun KS (2012) All-polymer hair structure with embedded three-dimensional piezoresistive force sensors. Sens Actuat A Phys 188:89-94

13. Pyo S, Lee Jl, Kim MO, Chung T, Oh Y, Lim SC, Park J, Kim J (2014) Development of a flexible three-axis tactile sensor based on screen-printed carbon nanotube-polymer composite. J Micromech Microeng 24:075012
14. Hwang ES, Seo JH, Kim YJ (2007) A polymer-based flexible tactile sensor for both normal and shear load detections and its application for robotics. J Microelectromech Syst 16:556-563

15. Ahmed M, Chitteboyina MM, Butler DP, Celik-Butler Z (2013) MEMS force sensor in a flexible substrate using nichrome piezoresistors. IEEE Sens J 13:4081-4089

16. Pyo S, Choi J, Kim J (2018) Flexible, transparent, sensitive, and crosstalkfree capacitive tactile sensor array based on graphene electrodes and air dielectric. Adv Electron Mater 4:1700427

17. Jung HI, Kwon DS, Kim J (2017) Fabrication and characterization of monolithic piezoresistive high-g three-axis accelerometer. Micro Nano Syst Lett $5: 7$

18. Eltaib MEH, Hewit JR (2003) Tactile sensing technology for minimal access surgery-a review. Mechatronics 13:1163-1177

\section{Publisher's Note}

Springer Nature remains neutral with regard to jurisdictional claims in published maps and institutional affiliations.

\section{Submit your manuscript to a SpringerOpen ${ }^{\circ}$ journal and benefit from:}

- Convenient online submission

- Rigorous peer review

- Open access: articles freely available online

- High visibility within the field

- Retaining the copyright to your article

Submit your next manuscript at $\mathbf{s p r i n g e r o p e n . c o m ~}$ 